\title{
Drenagem linfática no pós-operatório em lipoaspiração
}

A Lipoaspiração realizada para procedimentos estéticos tem o objetivo de melhorar a forma e eliminar as gorduras localizadas que são difíceis de serem removidas apenas com treinos e dieta, não é indicada para perde peso, sendo assim é indicada para indivíduos que se encontram próximo do seu peso ideal. Nas últimas três décadas, a lipoaspiração vem sendo aperfeiçoada, porém, como qualquer procedimento cirúrgico, não é isenta de complicações. A lipoaspiração consiste na remoção cirúrgica de gordura subcutânea, realizada por meio de cânulas submetidas a uma pressão negativa e introduzida por pequenas incisões na pele, pode ser considerada um dos maiores avanços dos últimos tempos, sendo hoje em dia, um dos procedimentos mais utilizados para que se consiga o corpo considerado perfeito. Mas para alcançar um resultado estético mais satisfatório, é necessária uma preocupação com os cuidados do pré e pós-operatório, acelerando a recuperação e prevenindo complicações mais comuns.

Palavras-chave: Drenagem Linfática Manual; Lipoaspiração; Pós-Operatório.

\section{Lymphatic drainage in the postoperative period in liposuction}

The Liposuction performed for aesthetic procedures aims to improve the shape and eliminate localized fats that are difficult to be removed only with training and diet, it is not indicated for losing weight, so it is indicated for individuals who are close to their weight ideal. In the last three decades, liposuction has been improved, however, like any surgical procedure, it is not without complications. Liposuction consists of the surgical removal of subcutaneous fat, performed through cannulas subjected to negative pressure and introduced by small skin incisions, can be considered one of the greatest advances of recent times, being nowadays one of the most used procedures for to achieve the body considered perfect. But to achieve a more satisfactory aesthetic result, a concern with pre and post-operative care is necessary, accelerating recovery and preventing more common complications.

Keywords: Manual lymphatic drainage; Liposuction; Postoperative.

Topic: Enfermagem Geral

Reviewed anonymously in the process of blind peer

Euzivane Pereira dos Santos

Instituto de Excelência em Educação e Saúde, Brasil

euzivanesantos@gmail.com

Marizete dos Santos Lima

Instituto de Excelência em Educação e Saúde, Brasil

euzivanesantos@gmail.com
Received: 20/02/2020

Approved: 15/06/2020
Referencing this:

SANTOS, E. P.; LIMA, M. S.. Drenagem linfática no pós-operatório em lipoaspiração. Medicus, v.2, n.2, p.30-36, 2020. DOI: http://doi.org/10.6008/CBPC2674-6484.2020.002.0005 


\section{INTRODUÇÃO}

Atualmente a busca constante pelo corpo perfeito cada vez veem se tornando uma obsessão, um sonho desejável aos padrões de beleza mais exigentes tanto para homens como mulheres. Motivo esse que inúmeras pessoas recorrem às cirurgias plásticas estéticas. Sabemos da existência de inúmeros procedimentos cirúrgicos utilizados para definir contornos e modelar o corpo da maneira mais desejada, entre eles a lipoaspiração, que buscam através dela obter o corpo dos sonhos.

Segundo Mauad (2008) "o surgimento de uma nova técnica conhecida como lipoaspiração representou um dos principais avanços da cirurgia plástica, em particular a do contorno corporal”. Essa técnica tornou-se a mais utilizada pelos cirurgiões na retirada de tecido adiposo melhorando o contorno do corpo e satisfazendo as expectativas das pacientes.

Sabe-se que após a lipoaspiração, o pós-operatório deve ter um tratamento especifico que auxilie o paciente a ter uma boa recuperação, tornando o resultado ainda mais satisfatório possível. Sendo a drenagem linfática uma técnica de grande eficiência e eficácia no pós-operatório de pacientes submetidos a procedimentos cirúrgicos.

Silva et al. (2012) afirma que a eficiência de uma cirurgia plástica não depende somente do seu plano cirúrgico, mas também dos cuidados pré e pós-operatório, que são fatores preventivos de possíveis complicações e promovem um resultado estético mais satisfatório. Este artigo tem como objetivo principal relatar os benefícios e a eficácia da drenagem linfática no pós-operatório imediato de lipoaspiração, sendo indicada por cirurgiões, lembrando que essa técnica deve ser aplicada por profissionais habilitados e com conhecimento da terapia ou técnica, que são muito utilizadas na recuperação de cirurgias plásticas estéticas.

\section{METODOLOGIA}

A pesquisa foi realizada em bases literárias e eletrônicas. A pesquisa foi limitada à língua portuguesa e no período de outubro a novembro 2019. As palavras chaves utilizadas foram: Drenagem Linfática Manual, Lipoaspiração e Pós-Operatório

\section{DISCUSSÃO TEÓRICA}

\section{Sistema linfático}

Segundo Fernandez et al. (2001), o sistema linfático está presente em todas as regiões dos membros. A pele, o tecido subcutâneo, as aponeuroses, os músculos, os tendões, os ossos, as articulações, as cápsulas articulares e suas dependências ligamentares, os nervos e os próprios vasos são objetos de uma cobertura linfática. Esse sistema retira o líquido acumulado no espaço intersticial e o leva de novo ao sangue por meio de drenagem dos dutos linfáticos, fluindo pelo canal torácico, junto às junções venosas da veia jugular interna e subclávia bilateralmente.

Lange (2012) afirma que todas as definições propostas sobre o sistema linfático, a mais clara, traz que o sistema linfático é uma via secundária de acesso, onde os líquidos advindos do interstício são entregues 
de volta ao sangue, ela tem uma forte ligação com os vasos sanguíneos e com os líquidos teciduais, que são esses absorvidos e transportados pela rede extensa dos capilares linfáticos e de vasos de grande calibre, desembocando pelo coletor principal no sistema nervoso.

\section{Capilares Linfáticos e Linfonodos}

Temos conhecimento de que o sistema linfático é formado, ou seja, composto por linfáticos iniciais que produzem a linfa, e pelos vasos linfáticos que conduzem a linfa. Sendo esses linfáticos iniciais as principais estruturas do sistema linfático, tendo como função principal absorver as macromoléculas.

Para Galvão (2005), os capilares linfáticos e venosos andam juntos, exceto no sistema nervoso central, em ossos, placenta, bulbo do olho e medula óssea. Os capilares formam os vasos linfáticos aferentes, que seguem pareados até atingir o linfonodo em sua porção cortical, deste saem os vasos linfáticos eferentes através do hilo, esses vasos têm o calibre maior e em número menor e que se dirigem a outro linfonodo.

Godoy et al. (2004), os linfonodos são barreiras limitantes, com a função de "filtrar" o sistema, sendo limitadores da velocidade de drenagem. Devemos obedecer à condição de filtração dos linfonodos, tendo um controle da velocidade da drenagem e da pressão exercida. Se devido a algum fator essa barreira seja forçada, tem risco de lesar os linfonodos. Naturalmente a linfa percorre por três a quatro linfonodos até atingir o sistema nervoso. Temos grupos de linfonodos na região axilar, virilha, pescoço, perna e em várias regiões profundas do corpo. Sendo assim, as várias técnicas que visam incrementar o fluxo linfático devem seguir sempre o sentido natural da drenagem, percorrendo os diferentes segmentos (GUIRRO et al., 2004).

\section{Linfa}

Para Borges (2006), a linfa derivada do líquido intersticial que flui para os linfáticos. Por isso logo após entra nos linfáticos terminais, ela apresenta a mesma composição que o líquido intersticial. Segundo Guirro et al. (2006) sabe-se que a linfa absorvida nos capilares linfáticos é transportada para os vasos coletores passando através de vários linfonodos sendo filtrada e recolocada novamente na circulação até atingir os vasos sanguíneos. Toda a linfa do organismo retorna ao sistema vascular sanguíneo através do ducto torácico e o ducto torácico direito. Sendo assim o ducto torácico recebe a linfa proveniente dos membros inferiores, do hemitronco esquerdo, do pescoço e da cabeça e do membro superior esquerdo, originando-se na cisterna do quilo, uma dilatação situada anteriormente à segunda vértebra lombar, onde desembocam os vasos que recolhem o quilo intestinal. Lembrado que o ducto torácico direito recolhe a linfa proveniente do membro superior direito, do hemotórax direito, do pescoço e da cabeça, formado pela união dos troncos subclávio, jugular e broncomediastinal direito.

\section{Drenagem linfática manual}

O médico dinamarquês Emil Vodder em 1936, criou a drenagem linfática tendo como uma técnica coadjuvante, estabelecida como padrão ouro no tratamento de linfedema. Entretanto, sabemos que atualmente a sociedade dita um padrão de beleza no qual muitas mulheres submetem-se a tratamentos sem 
fundamentação científica para se sentirem bem. A partir desta realidade, a drenagem linfática manual tornou-se uma técnica popular, realizada por pessoas sem conhecimento sobre a fisiologia e a fisiopatologia do sistema linfático e sua relação com demais sistemas no nosso organismo, e amplamente aplicada em indivíduos saudáveis para redução de medidas.

Sabe-se que o sistema linfático tem papel crucial no equilíbrio do fluído corporal e macromolecular, absorção lipídica e na função imune. Segundo Piccinin et al. (2011) a DLM está apresenta principalmente pelas técnicas de Vodder e Leduc. A diferença entre elas está no tipo de movimento. Vodder utiliza movimentos circulares, rotatórios e de bombeio, já Leduc propõe movimentos mais restritos. Todas as manobras, basicamente, constam de três fases: a primeira é a de apoio da mão e dos dedos sobre a pele da paciente, seguido pela fase ativa, que é a de empurrar o fluido; a terceira é a fase de repouso na qual a pele volta sozinha a sua posição inicial.

O tratamento no pós-operatório tem o objetivo de diminuir o edema e auxiliar no processo de cicatrização, na redução das áreas anestesiadas, na tonificação muscular e na microcirculação. Segundo Alencar et al. (2010), uma drenagem linfática bem-feita é capaz de alcançar os mais diversos resultados como otimização de resultados de pós-operatório, tratamentos e preparação para todas as cirurgias estéticas.

\section{Indicação e contraindicação da Drenagem Linfática Manual}

Sabe-se que o sistema linfático tem como uma das principais funções a drenagem dos líquidos que circulam no corpo, ocorre um acúmulo desses fluidos, pode surgir um edema, um dos sinais de um problema bastante desconfortável, que é o linfedema. Segundo Guirro et al. (2004) a drenagem linfática deve ser indicada de acordo com seus efeitos e disfunções relatadas pelo paciente, como: cicatrizes aderentes, hematomas, edemas, dor, diminuição da amplitude de movimento, tensão muscular e existem algumas doenças em que a drenagem linfática é contra indicada, como: Infecções agudas, insuficiência cardíaca descompensada, tromboses, flebite, tromboflebites, síndrome seno carotídeo, hipertensão, certos tipos de afecções da pele, câncer, asma brônquia e bronquite asmática, hipertireoidismo, reações alérgicas agudas, insuficiência renal dependente de diuréticos ou diálise

Para Migotto et al. (2013) a drenagem linfática manual é indicada em quase todo tipo de pósoperatório de cirurgia plástica, entre elas estão cirurgias de face, mamas, abdominoplastias, lipoaspiração, entre outras. Além disso, ela também é indicada em tratamentos de fibro edema gelóide, acnes, retenção hídrica, entre outros problemas. A drenagem deve ser bem-feita pelo profissional, pois assim ela melhora o quadro de dor, pois diminui a congestão do tecido, favorecendo assim o retorno mais rápido da sensibilidade cutânea local. Além disso, a drenagem linfática não causa nenhum tipo de risco à saúde do paciente e pode ser usada repetidas vezes sem acarretar problemas.

\section{Benefícios da Drenagem linfática Manual}

Sabemos que a drenagem linfática manual (DLM) tem como principal objetivo a captação, reabsorção e evacuação da linfa, essa técnica é realizada com pressões suaves, lentas, intermitentes e relaxantes. A DLM 
é um método que mobiliza a linfa, retirando o acúmulo de líquidos de regiões corporais onde estas se acumulam, resultando na melhora da oxigenação e circulação do local.

São inúmeros os seus benefícios, sendo indicada na redução de edemas e linfedemas, fibro edema gelóide, insuficiência venosa crônica, cefaleias, edemas gestacionais, síndrome pré-menstrual, fadiga, dores nas pernas, irritabilidade, ansiedade, pré e pós-cirurgias. Segundo Alencar et al. (2010), uma drenagem linfática bem-feita é capaz de alcançar os mais diversos resultados como otimização de resultados de pósoperatório, tratamentos e preparação para todas as cirurgias estéticas.

\section{Manobras da drenagem linfática}

Segundo a técnica baseada em Leduc, fala sobre a utilização de cinco movimentos combinados entre si formando um sistema de massagem, são eles: A drenagem dos linfonodos, onde faz necessário conhecer suas localizações iniciando a massagem pelo contato direto dos dedos indicador e médio sobre os linfonodos, essa manobra é realizada com uma pressão de nível moderada e rítmica; com movimentos circulares onde é utilizado todos os dedos da mão do terapeuta, sendo leves e rítmicos, continuando sempre o sentido da drenagem fisiológica; com movimentos circulares realizados somente com o polegar; os movimentos combinados, sendo feitos movimentos circulares hora com todos os dedos da mão, hora apenas com o polegar; e pôr fim a técnica conhecida como bracelete, utilizada quando o edema atinge grandes áreas, a manobra pode ser realizada pelo terapeuta uni ou bimanual, e o sentido deve ser distal para proximal, obedecendo o sentido da drenagem fisiológica e a pressão deve ser intermitente.

Já a técnica de acordo Emill Vodder, propõe quatro tipos de movimentos, sendo eles: círculos fixos, colocando a mão espalmada sobre a pele fazendo movimentos circulares com os dedos promovendo um estiramento do tecido; movimentos de bombeamento, onde são realizadas pressões decrescentes da palma da mão para os dedos de forma intermitente; movimento do doador, que é feito com as palmas das mãos realizando um movimento de arraste seguido de uma pronação do antebraço; A técnica de movimento giratório ou de rotação, que é realizado com a face palmar deslizando sobre a pele do paciente fazendo um desvio ulnar. Os movimentos de todas as técnicas devem ser rítmicos, alternados e unidirecionais, sempre seguindo o sentido da drenagem fisiológica

\section{A eficácia de realizar a drenagem linfática no pós-operatório de lipoaspiração}

Para Mauad (2008), os sintomas causados pelo pós-operatório podem ser minimizados, principalmente quando houver um atendimento profissional utilizando como conduta a drenagem linfática manual. Isso faz com que o hematoma, edema diminua rapidamente promovendo a formação de uma nova vascularização, o que diminui e previne a formação de retrações, queloides e cicatrizes hipertróficas.

No estudo realizado pelo autor Tacani et al. (2005), a DLM é uma técnica de massagem designada a promover a melhora das funções do sistema linfático, através de manobras precisas, lentas, rítmicas e leves, que percorrem o trajeto dos vasos linfáticos presente superficialmente, onde empurra tangencialmente a pele, sem ocasionar alguma friç̧ão, deslizamento, dor ou eritema. É considerada um dos métodos mais 
válidos no pós-operatório de cirurgias plásticas promovendo uma melhor reabsorção de edemas, ela auxilia no processo de reparação do tecido devida o fibrinogênio da linfa, prevenindo possíveis fibroses e aderências, gerando uma renovação dos capilares linfáticos que foram lesionados e promove um efeito analgésico.

\section{Lipoaspiração e suas técnicas}

Segundo Guirro et al. (2004), as primeiras tentativas em aplicação do método da lipoaspiração ocorreram em 1920, quando foi utilizada uma curetagem na região de quadril para retirada de gordura sem necessidade de fazer ressecção nos tecidos, essa tentativa não apresentou sucesso, levando a uma necrose e amputação por uma infecção e lesão vascular. O primeiro método usando cânulas de aspiração ligadas ao aspirador motorizado foi na década de 70, que permitia o deslocamento simultâneo com a lipossucção. De acordo com Sabatovich (2009), no mês de novembro de 1980, foi realizada a primeira cirurgia de lipoaspiração no Brasil, feita por Lllouz que utilizou uma cânula de $12 \mathrm{~mm}$.

As cânulas mais grossas aspiram rapidamente, mas causam maior traumatismo tecidual, com maior sangramento, já as cânulas mais finas demoram mais para remover a mesma quantidade de gordura, mas causam menos traumatismo aos tecidos, com menor sangramento. A cânula se move rapidamente para frente e para trás para retirar as células gordurosas, que são sugadas para fora. Devem ser realizados movimentos de varredura, na área a ser tratada, de preferência se cruzando perpendicularmente para evitar ondulações e irregularidades. A Lipoaspiração é um procedimento que traumatiza a região por onde o tubo passa. Esse traumatismo provoca sangramento e edema, por isso é prudente não se fazer retiradas maiores do que dois litros de volume aspirado.

\section{Complicações no pós-operatório de lipoaspiração}

Pinto (2011), relata que é procedimento cirúrgico de lipoaspiração causa uma lesão tecidual, a qual atinge os tecidos epitelial e conjuntivo, que em resposta iniciam o processo de cicatrização. Constatou-se que a fibrose é presente em grande parte do pós-cirúrgico de lipoaspiração e está relacionada à cicatrização, que é apresentada por sinais como: diminuição da extensibilidade da pele, tecido endurecido, irregular e doloroso. Isso gera sobretudo, um incômodo estético, ao paciente: a fibrose é facilmente observada e palpada, e vários estudos como manifestação principalmente em paciente submetidas à lipoaspiração. Nas complicações locais, o maior índice são as irregularidades na pele, que são visíveis e palpáveis, a presença de edema prolongado, equimoses hiperpigmentação, hematomas, seromas, fibrose, aderência, diminuição da sensibilidade, necrose, infecções locais, dermatites de contato, cicatrizes inestéticas. Nas complicações de ordem sistêmica da lipoaspiração apresentam-se perfurações de vísceras, reações alérgicas diante de algum medicamento utilizado no intra e pós-operatório, febre, infecção sistêmica, arritmias cardíacas, taquicardias, anemia, tromboembolismo pulmonar, choque hipovolêmico (FRANCO et al., 2011). Segundo Migotto et al. (2013), na maioria dos casos, essas complicações podem ser evitadas, pela correta indicação do médico para a cirurgia, e cuidados com os princípios técnicos que a envolve. Há também os cuidados específicos, tanto no período pré-operatório quanto no pós, que devem ser tomadas não só pelo médico, mas por todos que estão 
acompanhando o paciente.

\section{CONCLUSÕES}

Atualmente vivemos numa sociedade em que as mídias estão mais exigentes com as pessoas em relação ao corpo, exigindo cada vez mais um corpo escultural, essas medidas exageradas estão cada vez mais explícitas nas mídias. As pessoas sempre na busca do corpo ideal estão cada vez mais disputadas, e as demandas para procedimentos cirúrgicos as estatísticas de cirurgias plástica só aumentam cada vez mais.

Sendo assim a drenagem linfática é uma técnica realizada por profissionais habilitados que apresentam grande eficácia nos pré e pós-operatório de lipoaspiração, para diminuição do edema, redução no quadro da dor, proporcionando vários benefícios e conforto aos pacientes após as sessões e parar que obtenham melhores resultados ao paciente, quanto mais precoces o início do tratamento mais rápido é sua cicatrização e recuperação, onde todos esses objetivos da drenagem linfática proporciona aos pacientes submetidos a cirurgia plástica. Sendo assim, esse artigo apresenta os benefícios a eficácia da drenagem linfática no pré e pós-operatório de lipoaspiração.

\section{REFERÊNCIAS}

ALENCAR, T. P.; MEJIA, D. P. M.. A influência da drenagem linfática manual no pós-operatório imediato de cirurgia vascular de membros inferiores. 2010.

BORGES, F.. Dermato-funcional: modalidades terapêuticas nas disfunções estéticas. São Paulo: Porte, 2006.

FERNANDES, J.; SOUZA, A.. A ação da drenagem linfática manual no pós-operatório de abdominoplastia. 2001.

FRANCO, F. F.; BASSO, R. C. F.; TINCANI, A. J.; KHARMANDAYAN, P.. Complicações em Lipoaspiração clássica para fins estéticos. Revista Brasileira de Cirurgia Plástica, v.1, n.27, p.135-140, 2011.

GALVÃO, M. M.. Drenagem Linfática Manual e Ultrassom no tratamento do fibro edemageloide em região glútea: um estudo de caso. Monografia (Bacharelado) - Faculdade Assis Gurgacz, Toledo, 2005

GODOY, J.; GODOY, M.. Drenagem linfática manual: novo conceito. Rev. Vasc. Br., v.3, n.1, 2004

GUIRRO, E.; GUIRRO, R.. Fisioterapia dermato-funcional: fundamentos, recursos e patologias. São Paulo: Manole, 2006. p.73-80.

GUIRRO, E.; GUIRRO, R.. Fisioterapia dermato-funcional: fundamentos, recursos, patologias. 3 ed. São Paulo: Manole; 2004.
LANGE, A.. Drenagem linfática no pós-operatórios das cirurgias plásticas. 22 ed. Curitiba: Vitória, 2012.

MAUAD, R.. Estética e cirurgia plástica: tratamento no pré e pós-operatório. 3 ed. São Paulo: SENAC, 2008.

MIGOTTO, J. S.; SIMÕES, N. D. P.. Atuação fisioterapêutica dermato funcional no pós-operatório de cirurgias plásticas. Revista eletrônica Gestão e Saúde, Florianópolis, v.4, n.1, 2013

PICCININ, A. M.; MELLO, P. B.; BEM, D. M.; SILVA, A.; ROSA, P. V.. Redução do edema em membros inferiores através da drenagem linfática manual: um estudo de caso. Revista Eletronica Inspirar, 2019.

PINTO, A. B.. Avaliação dos níveis de fibrose decorrente do pós-operatório de cirurgias plásticas. Revista Iniciação Cientifica, Belo Horizonte, v.11, 2011.

SILVA, R. M. V.; SILVA, L. M.; RAMOS, M. L. V. S.; SILVA, A. C. F.; MEYER, P. F.. Investigação sobre o encaminhamento médico aos tratamentos fisioterapêuticos de pacientes submetidos a cirurgia plástica estética. Cadernos da Escola de Saúde, Curitiba, v.2, n.8, 2012.

TACANI, R. E.; ALEGRANCE, F. C.; ASSUMPÇÃO, J. D’A.; GIMENES, R. O.. Investigação do encaminhamento médico a tratamentos fisioterapêuticos de paciente submetidos a lipoaspiração. O Mundo da Saúde, São Paulo, v.29, n.2, p.192-98, 2005.

A CBPC - Companhia Brasileira de Produção Científica (CNPJ: 11.221.422/0001-03) detém os direitos materiais desta publicação. Os direitos referem-se à publicação do trabalho em qualquer parte do mundo, incluindo os direitos às renovações, expansões e disseminações da contribuição, bem como outros direitos subsidiários. Todos os trabalhos publicados eletronicamente poderão posteriormente ser publicados em coletâneas impressas sob coordenação da Cognitionis Publishing, da Companhia Brasileira de Produção Científica e seus parceiros autorizados. Os (as) autores (as) preservam os direitos autorais, mas não têm permissão para a publicação da contribuição em outro meio, impresso ou digital, em português ou em tradução. 